\section{Carbon Nanotube Membranes Enhance Desalination}

\section{The Pitch}

The water supplies of the world face great stresses due to increasing populations, pollution, and drought. There is a need for an efficient means of filtering and desalinating water that does not use excessive amounts of energy and can provide highly effective filtration of both chemicals and biological organisms. The scale of the problem is enormous: approximately 1 billion people do not have access to clean water, more than 2 billion people now live in water-stressed areas, and this number could climb to 3.5 billion by 2025 . The combination of water desalination and purification is one way to alleviate this problem. Reverse osmosis is the present most efficient desalination method. However, a typical seawater reverse-osmosis plant still requires more than $10 \mathrm{Wh}$ of electricity to produce $1 \mathrm{gal}$ of fresh water.

A novel membrane architecture (shown in Figure 1) composed of carbon nanotubes (CNT) with pores smaller than $2 \mathrm{~nm}$ that could exclude ions and other particles has been developed by Lawrence Livermore National Laboratory (LLNL). This membrane technology could help to address the urgent worldwide need for simple, energyefficient ways to produce clean water. Livermore's membranes have the potential to improve the desalination process by significantly reducing the membrane's flow resistance. The unique nature of the carbon nanotube pores supports a much higher water flux for the same applied pressure relative to conventional membrane pores. Molecular dynamics simulations demonstrate that this effect is due to the highly slippery nature of the nanotube walls as well as to the formation of chains of water molecules in the extremely narrow nanotube channels.

Growing water scarcity and cost reductions in employing reverse osmosis have increased demand for membrane-based desalination. The business research firm Frost and Sullivan estimates that the water desalination plant market in the United States was worth $\$ 537$ million in 2006 and is growing at a 7\% compound annual growth rate. Reducing two primary cost drivers-capital costs and energy consumption-is expected to make the

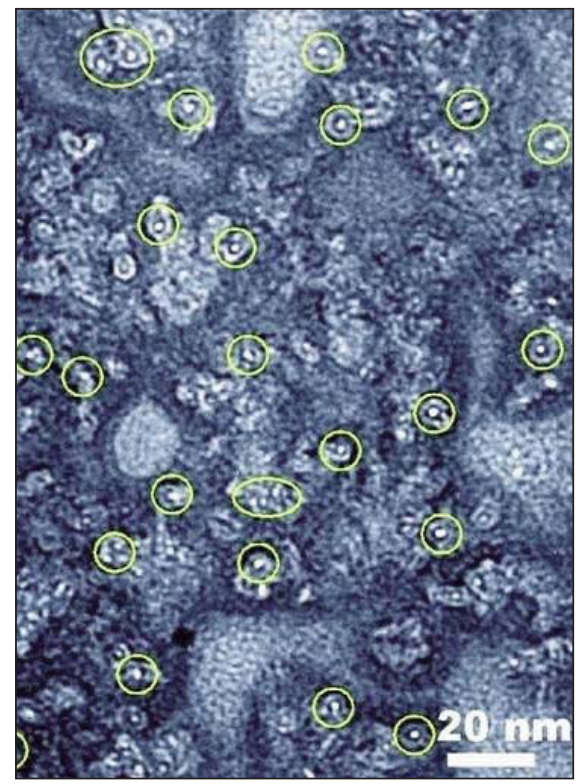

Figure 1. Transmission electron micrograph of a carbon nanotube membrane cross-section that shows sub-2-nm membrane pores.

greatest impact on the reverse osmosis (RO) desalination industry. Reduction in the costs of RO systems will increase the growth of the market, in both developed and emerging countries.

In addition, carbon nanotube pores have potential uses besides desalination. Biological pore channels share a number of structural and functional features with CNTs that make CNT nanofluidic platforms ideal candidates for the realization of a robust, biomimetic system that could exploit the fast transport, selectivity, and gating properties of biological pores. Possible applications range from controlled nanoscale delivery of therapeutics to molecular sensing.

TECHNOLOGY ADVANCES seeks materials developments on the threshold of commercialization. Send suggestions to Renée G. Ford, Renford Communications, renford@comcast.net.

\section{The Technology}

LLNL's nanofluidic platform consists of smaller than 2-nm diameter CNT membranes fabricated by a conformal coating of a dense vertically aligned CNT forest. Conformal encapsulation of the nanotubes by low-pressure silicon nitride deposition produces a gap-free matrix that fills the volume between the doublewalled CNTs. Argon ion milling then removes the excess silicon nitride on both sides of the membrane and the catalyst particles. Reactive ion etching in oxygencontaining plasma further exposes and opens the CNTs ends. The etching processes, used to expose and selectively uncap the CNTs, introduce hydroxyl, carbonyl, and carboxylic functional groups at the nanotube entrance. In particular, ionization of these carboxylic groups provides a ring of negative charges at the pore entrance that affects ion transport through the nanotube pore. Recent research by the LLNL group established that the ion rejection due to this charge at the mouth of the CNT pore is governed by the Donnan membrane equilibrium theory, which states that the observation that charged molecules starting on one side of a semipermeable membrane sometimes will not evenly distribute themselves by diffusion on both sides of the membrane. This effect probably occurs because there are other charged substances already present that cannot move through the membrane themselves and which are creating an electric field that influences the movement of the incoming charged molecules.

\section{Opportunities}

The researchers and LLNL are seeking development partners for their carbon nanotube membrane technologies.

Source: For technical information, Olgica Bakajin, Lawrence Livermore National Laboratory, P.O. Box 808, Livermore, CA, 94551, USA; 925-4220931; and email bakajin1@1lnl.gov; or Aleksandr Noy, 925-424-6203; and e-mail noy1@1lnl.gov. For business development, investment, or licensing: Ida Shum, Industrial Partnerships Office, Lawrence Livermore National Laboratory, Livermore, CA, 94551, USA; 925-423-9724; and e-mail shum3@1lnl.gov.
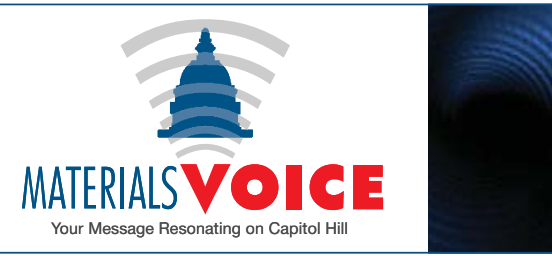

A Web-based tool to ensure that your voice is heard on Capitol Hill www.mrs.org/materialsvoice 